ORIGINAL ARTICLE

\title{
School hearing screening programme in the UK: practice and performance
}

\author{
S Fonseca, H Forsyth, W Neary
}

See end of article for

authors' affiliations

Arch Dis Child 2005;90:154-156. doi: 10.1136/adc.2003.046979

......................

Correspondence to:

Dr S Fonseca, Clare

House, St George's

Hospital, London SW17

OQT, UK; Sarita.Fonseca@

stgeorges.nhs.uk

Accepted 15 May 2004

\begin{abstract}
Background: Paediatric audiology services and screening programmes are currently under review.
Aims and Methods: To investigate current practice and performance of the school hearing screening programme (SHSP) by means of a questionnaire.

Results: SHSP was found to detect previously unrecognised hearing loss at low cost. Wide variation in practice was shown, and the majority of services had no computerised system for data collection. Conclusion: There is a need for nationally agreed protocols and quality assurance procedures.
\end{abstract}

M ost districts in the UK offer a school hearing screening programme (SHSP). ${ }^{1}$ However, the purpose of SHSP has been questioned. ${ }^{2}$ Is the aim to detect undiagnosed sensorineural hearing impairment (SNHI), or conductive hearing impairment (CHI), or both?

Despite recommendations for the retention of the SHSP and standardisation of test protocols, ${ }^{3-6}$ there is concern regarding the lack of uniformity in services provided nationally.

At a time when services are undergoing major change and when screening programmes are at the forefront of service policy considerations, it is appropriate to evaluate current practice.

\section{METHODS}

This questionnaire based study relating to the SHSP for the academic year September 2000 to August 2001 was carried out by the British Association of Community Doctors in Audiology (BACDA). BACDA members were invited to contribute to the study. Non-BACDA members were included by instructing the membership to pass study details to the designated person responsible for SHSP.

Data were collected on:

- Screening protocol, including target population, test procedure, and personnel involved

- Coverage, referral rate, and yield

- Cost.

Further information was requested from those who reported that no screen was carried out in their area, and from those who were unable to access relevant data.

\section{RESULTS}

Replies were received from 96 services: a response rate of $70 \%$. Eighty two services agreed to contribute to the study, four $(4.2 \%)$ had no SHSP, and $10(10.4 \%)$ opted out, as they were unable to access data. During the study period it became apparent that data collection was more difficult than anticipated. As some clinicians were unable to access data relating to some areas of the study, results, when expressed as percentages, relate to number of responses received for that area of study.
All services screen children in mainstream state schools. There is a variable approach to the screening of children in private schools and special schools. The majority of children $(87.5 \%)$ are screened in their 1 st or 2 nd year at school.

Eight categories of professionals conduct screening tests, the majority of services employing school nurses or audiometric screeners.

A two stage screening procedure is carried out by 64 (79\%) services, a one stage procedure by $17(21 \%)$. Audiometric test procedures vary both in technique, frequencies, and levels used for screening. Seven services used middle ear function tests, mainly during the second stage. There was no reported use of screening questionnaires.

Following an unsatisfactory screen, most children are referred to community audiology clinics (67.6\%) and GPs $(21 \%)$. Others are referred directly to ENT.

Four units had discontinued screening due to "financial constraints", "local audit confirming low yield", and "lack of cost effectiveness".

Of the 82 service units initially agreeing to contribute to the study, $39(47.6 \%)$ found, during the study period, they were unable to access relevant information. Forty three units supplied information, which in some cases was incomplete.

Eligible population data were obtained from education and health sources using both manual and computerised records. The total eligible population was reported as 125 687; the number of children screened was 109505 , giving a mean coverage of $87 \%$. The overall yield was $2.9 \%$ and the yield of new cases was $2.2 \%$. Figure 1 shows the detailed outcome for children screened.

Forty nine of 96 respondents were unable to provide information on coverage, referral rates, and yield due to staff shortages and lack of robust data collection systems.

Costs, including pay (annual pay and employer's cost), equipment, training, and overheads (accommodation, travel, heating, lighting, and consumables) were calculated. The cost per child screened was less than $£ 10$ in 19 units, $£ 10-£ 20$ in nine units, and more than $£ 20$ in four units. The average cost per test was $£ 9.90$. Considering newly identified cases in this study, regardless of severity or permanence of impairment, the cost per child identified was $£ 460$.

Abbreviations: $\mathrm{CHI}$, conductive hearing impairment; SHSP, school hearing screening programme; $\mathrm{SNHI}$, sensorineural hearing impairment 


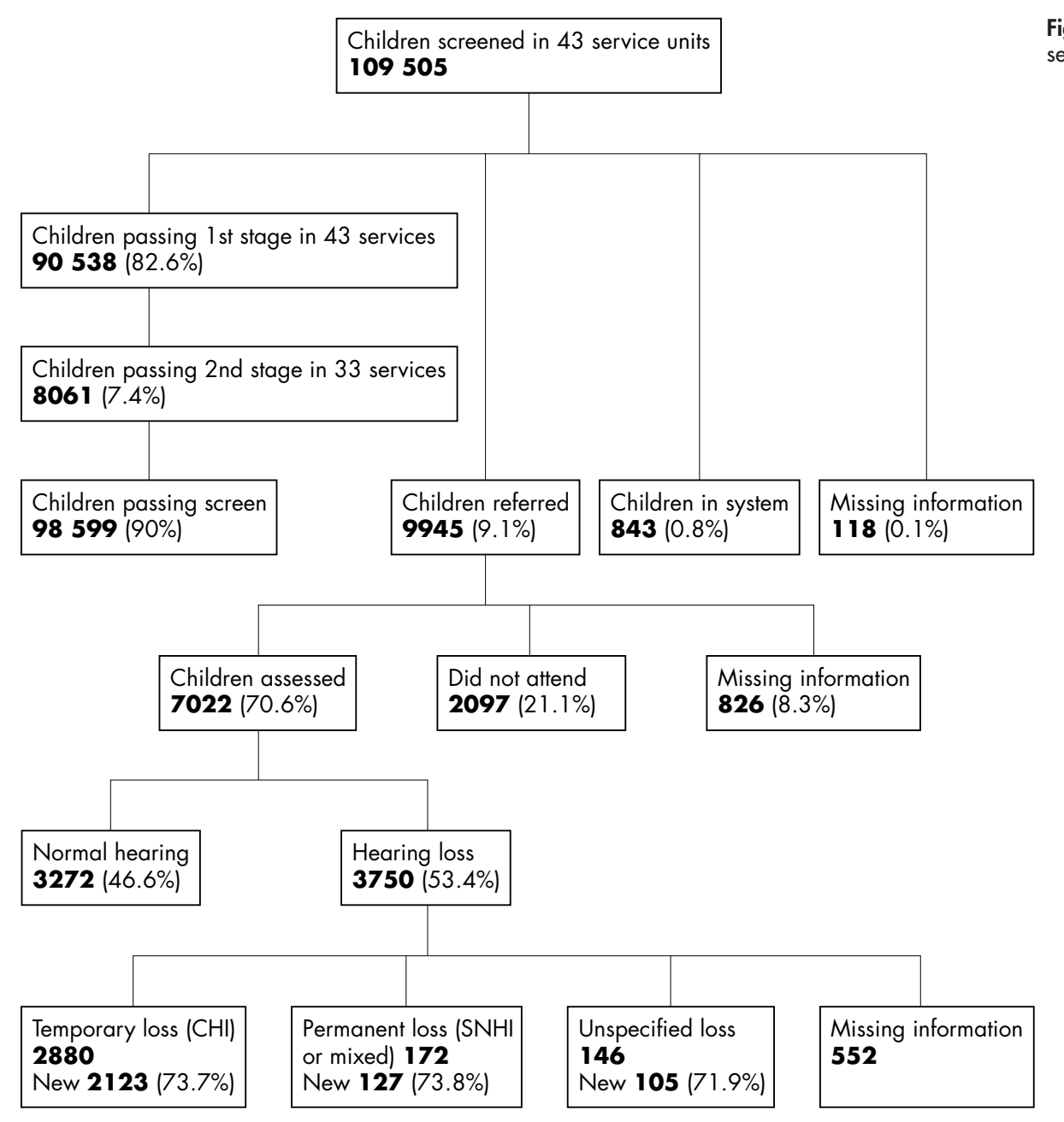

Figure 1 Outcome (data from 43 services).

\section{DISCUSSION}

Practice described in this study is based on data from $70 \%$ of service leads invited to participate. The distribution of responding services suggests our findings are geographically representative of services across Scotland, England, Wales, and Northern Ireland in urban and rural areas. However, given the frequent restructuring of the health service, it is difficult to comment on the representative nature of the sample other than on a geographical basis.

Despite recommendations, in the absence of national guidelines, services have developed in response to local circumstances. In recent years, revision of the School Health Service, together with the evolving role of the community paediatrician has resulted in many changes. Routine medical inspections are no longer carried out, but the SHSP remains widely available across the UK.

Variation in professional background of screeners does not of itself compromise quality. Adequate training and monitoring of techniques is more important than professional background. ${ }^{7}$

It is a cause for concern that more than half the responders were unable to provide information regarding coverage. It is unacceptable that a screening procedure involving large numbers of children and many professional man-hours is not adequately supported by information technology allowing routine monitoring of performance.

Where it has been possible to report on coverage, the results for some services were encouraging, although nationally significant improvements should be made. It is important that services look to improving their data collection systems in order to audit the screen.

As expected, following a universal screen of children in this age group, a substantial number were referred for further audiological assessment. The target age group is such that there will be children who are unwilling or unable to cooperate, necessitating further assessment. The prevalence of mild or transient conductive hearing loss can be expected to contribute significantly to those requiring further assessment, particularly during the winter months.

The non-attendance rate $(23 \%)$ is high, but similar to that for general paediatric outpatient clinics. ${ }^{8}$ As the screening test is frequently carried out in the absence of carers, consent procedures and information about the screening process should be reviewed to improve understanding of the process and encourage attendance following an unsatisfactory screen. Lack of understanding of the process, together with lack of parental concern, may contribute to failure to comply with appointments.

For the purpose of this study a true case is regarded as a child who requires ongoing supervision within audiology or ENT. This group consists of children whose management includes advice to parents, referral to education advisory services, watchful waiting, medical and surgical treatment, and amplification. A yield of $2.9 \%$ cases is shown, with $2.2 \%$ being newly identified as hearing impaired. These figures can be translated into approximately one child in every class screened. Screening children already known to services will not contribute to the child's medical management, other than 
those lost to the system. However, recognition of such children by SHSP will ensure that the classroom teacher is made aware. As would be expected, the majority of losses identified were due to temporary CHI, and permanent losses identified were mainly unilateral or of a mild or moderate degree.

There has been increasing suggestion from the literature, that mild, moderate, fluctuating, or intermittent hearing losses due to middle ear disease may adversely affect school performance and behaviour. ${ }^{10}$ Similarly there is more understanding about the effect of unilateral hearing loss. The question posed regarding the purpose of the SHSP in relation to SNHI and CHI needs further consideration in light of this evidence. It must be remembered that terms such as mild or minimal refer to hearing threshold levels and not to the effects on the child.

The average cost at $£ 9.90$ per child screened is low. The cost per child newly identified with any degree of temporary or permanent loss is $£ 460$. The value of hearing screening relates both to children confirmed as having normal hearing as they embark on formal education and to those found to have hearing impairment, hence our consideration of cost per child screened. Documentation of hearing levels at school entry provides useful information on children presenting with other difficulties early in their school lives, and also in children presenting later with hearing problems, although this is not a primary aim of the SHSP.

Introduction of the national newborn hearing screening programme (NHSP) across the UK may have some impact on yield, particularly regarding previously unrecognised unilateral hearing loss. However, it will be several years before all children entering school will have had their hearing screened in the newborn period. We suggest that it is appropriate to retain the SHSP during this interim period. There should be nationally agreed quality standards and guidelines regarding screen procedures and training requirements for screeners. Services should ensure that the screen is adequately resourced to allow staff involved to follow protocols and procedures and to adequately evaluate the programme.

It should be remembered that the efficiency of the SHSP and added value from the screen depends on close liaison between health services and education in terms of identifying children for testing and communicating results and management strategies to classroom teachers.

\section{Conclusions}

The study identifies wide variation in current practice and shows a need to consider protocols, test procedures, training, and quality assurance nationally. There should be further discussion and clarification about case definition.

Difficulty with staffing and data collection shows the need for adequate resources in line with technological advances. IT support is essential to allow monitoring and thus increase awareness of coverage and referral rates.

The SHSP is shown to identify children not previously recognised as having hearing impairment at low cost.

In the short term, the SHSP should continue. Following the implementation of NHSP there will be further discussion on the role of SHSP. Reconsideration of cost effectiveness may result in consideration of alternative methods of identifying children whose hearing loss may otherwise go undetected.

\section{ACKNOWLEDGEMENTS}

We acknowledge BACDA research group members for their part in planning this study; BACDA members and others who contributed by submitting data; Christine Waterman for statistical analysis, Dr V Hillier for statistical advice, and BACDA for financial support.

\section{Authors' affiliations}

S Fonseca, Consultant Community Paediatrician (Audiology), Clare House, St George's Hospital, London, UK

H Forsyth, Consultant Community Paediatrician (Audiology), Royal Liverpool Children's Hospital, Liverpool, UK

W Neary, Consultant Community Paediatrician (Audiology), Child \& Family Services Unit, Warrington, UK

Competing interests: none declared

\section{REFERENCES}

1 Davis A, Bamford J, Wilson I, et al. A critical review of the role of neonatal hearing screening in the detection of congenital hearing impairment. Health Technol Assess 1997;1:10.

2 Bamford J. Paediatric Audiology in the United Kingdom: moving off in several directions. Br J Audiol 1986;20:175-9.

3 ACSHIP. Final report of the subcommittee appointed to consider services for hearing impaired children. London: DHSS, 1981.

4 Haggard M. Screening children's hearing. Br J Audiol 1992;26:209-15.

5 Hall DMB, ed. Health for all children. A report of the Joint Working Party on Child Health Surveillance. Oxford: Oxford University Press, 1996.

6 Polnay L, ed. Health needs of school age children: Report of a Joint Working Party. London: British Paediatric Association, 1995.

7 Smith PA, Evans PIP. Hearing assessment in general practice, schools and health clinics: guidelines for professionals who are not qualified audiologists. Br J Audiol 2000;34:57-61.

8 McClure RJ, Newell SJ, Edwards S. Patient characteristics affecting attendance at general outpatient clinics. Arch Dis Child 1996;74:121-5.

9 Smith WR, Pither RE. Long term follow-up of children with mild hearing impairment: pre and post intervention. Journal of the British Association of Teachers of the Deaf 1993:4(17).

10 Tharpe AM, Bess FH. Minimal, progressive and fluctuating hearing loss in children: characteristics, identification and management. Paediatr Clin North Am 1999;46:65-78. 